\title{
DEMONSTRATION OF THE INVALIDITY OF THE MPEMBA EFFECT
}

\section{GIUSEPPE VERDE}

Student, Parthenope University of Naples, Italy

Demonstration of the invalidity of mpemba effect with thermodynamic processes and its operating method are described. The mpemba effect is a process, in which hot water can freeze faster than cold water. This assertion is counter-intuitive. Indeed, the mpemba effect breaches fundamental thermodynamic laws. In fact, that there is no evidence meaningful of the validity of the mpemba effect [1]:the phenomenon, when taken to mean "hot water freezes faster than cold", is difficult to reproduce or confirm because this statement is ill-defined; although the mpemba effect is real, it is unclear whether the explanation is trivial or enlightening. However, with these definitions, this effect has never been clear. On the other hand, there are many the circumstances in which the effect is not observed. The main references to the effect go back at least to the writings of aristotle [2], but the author shows that this effect is mistaken and shows the available evidence for negation of the mpemba effect and carry out experiments by cooling water in carefully controlled conditions. A mathematical demonstration has been presented, that violates the physical principle, coming to a contradiction of thermodynamic laws. The demonstration would show therefore, the mpemba effect cannot occur, and it does not exist if the initial parameters are correctly equivalents. The required vast multidimensional array of experiments explains why the effect is not yet understood and demonstrated.

Therefore, it is necessary to verify all the previous/historical relationships are the result of a bad experimental technique, since evidently it started from incorrect assumptions. It is important and necessary to stress that, in order to validate the thesis: all conditions must be the same at any time.
\end{abstract}

KEYWORDS: Thermodynamics, Mpemba, Coldest Water \& Hottest Water

Received: Aug 07, 2018; Accepted: Aug 27, 2018; Published: Sep 21, 2018; Paper Id.: IJAERDDEC20182

\section{INTRODUCTION}

To describe this phenomenon, in the past, some explanations had been proposed. However, the following observations studied are not sufficient to resolve the effect, including:

- Evaporation: The evaporation of the hottest water reduces the mass of the water to be frozen [3].

But, I will show that this thesis is not valid for the experiment observed. The reason is very simple: the evaporation of water would cause a decrease in mass. Therefore, the experiment cannot be considered valid, since the initial characteristics have changed. System's mass cannot change, so quantity cannot be added nor removed.

Anyway, if the mass differences are present and considered valid for the experiment observed, they are negligible, because don't significantly change the time needed for water to freeze.

- Convection: Accelerating heat transfers. In the warmer water, the convention motion improves the heat exchange with the external environment [4]. Higher convection in the warmer water may spread ice crystals around faster. This thesis, if supposed valid, has a limit. 
It is considered that:

$T_{h}=$ hottest water temperature.

$T_{c}=$ coldest water temperature

$t_{h}=$ freezing time for the hottest water

$t_{c}=$ freezing time for the coldest water

In this experiment, water is cooled to $0^{\circ} \mathrm{C}$ for two water samples. It is supposed that hottest water takes a $t_{1}$ time to switch from temperature $T_{h}$ to the $T_{c}$ temperature. Meantime, the coldest water assumes a temperature $T<T_{c}$. From the instant of time $t_{1}$, the hottest water will proceed at the same speed as the coldest water, but at a higher temperature. Therefore, the hottest water will reach the temperature of $0^{\circ} \mathrm{C}$ in a time $t_{h}>t_{c}$. Figure 1 shows the thermal effect explained through an asymptotic representation.

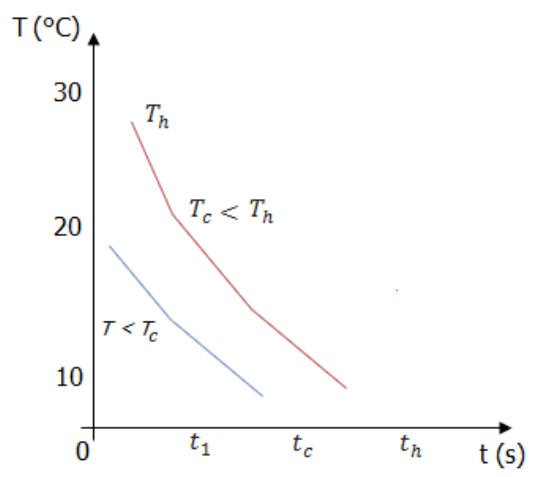

Figure 1: A Graph of Freezing Rates for the Two Water Samples

- Frost: It would have insulating effects. The coldest water would tend to freeze from the top, reducing further heat loss, while the hottest water would tend to freeze from the bottom.

Similarly, the previous demonstration for the convection process can also be used in this case.

- Thermal Conductivity: The hottest water can melt through a layer of frost that acts as an insulator, allowing the water to come into direct contact with a lower layer much colder. Therefore, the hottest water then cools much more quickly from this point on.

But also in this case, it is shown that this thesis is not valid for the experiment: the hottest water absorbs more thermal energy. Therefore, the experiment cannot be considered valid, since the initial conditions of the observations are different.

Finally, all the other cases are attributable to the previous demonstration for the convection process, among which:

- Dissolved Gases: Coldest water would contain more dissolved gases than the hottest water. This phenomenon would change the properties of the water with respect to convection currents.

- Hydrogen Bonding: In warm water, hydrogen bonding is weaker. 
- Crystallization: The hottest water might be responsible for the faster crystallization due to relatively higher population of hexamer states [5].

- Distribution Functions: from the Maxwell-Boltzmann distribution showing up in gases [6].

If these phenomena are present, they are considered valid in a limited time for the experiment observed.

Therefore, it is clear that the explanation is trivial. Although the Mpemba effect could be real, it is only the consequence of obvious characteristics.

\section{THEORETICAL REFERENCES}

Sensible heat is defined as heat exchanged by a body or thermodynamic system, which produce a decrease in the temperature difference, but leaving unchanged the other thermodynamic parameters, such as volume and pressure[7] [8] [9] [10].

The sensible heat $\left(Q_{S}\right)$ of a thermal process may be calculated as the product of the thermodynamic system mass (m) with its specific heat capacity $(\mathrm{c})$ and the change in temperature $(\Delta \mathrm{T})$ :

$$
\mathrm{Q}_{\mathrm{s}}=m c \Delta \mathrm{T}
$$

As a form of energy, heat $(\mathrm{Q})$ has the unit joule $(\mathrm{J})$ in the International System of Units (SI), whereas the standard International System of Units, (SI) unit of mass is the kilogram (kg). Heat capacity or thermal capacity is defined as the ratio of the heat added to or removed from a thermodynamic system to the resulting temperature change. The unit of heat capacity is joule per kelvin in the International System of Units (SI):

$$
\frac{\mathrm{J}}{K}
$$

The specific heat capacity or specific heat is the heat capacity per unit mass of a thermodynamic system:

$$
\frac{\mathrm{J}}{\mathrm{kgK}}
$$

In fact, in science and engineering, thermodynamic properties are often defined by the specific term [11] [12].

The term $Q_{s}$ is used differently with latent heat $\left(Q_{L}\right)$. Latent heat is the thermal energy released or absorbed by a thermodynamic system during an isothermal and isobaric process, involved respectively in phase changes at a specified temperature and pressure. It is defined as the latent heat of fusion for a substance condensing and latent heat of vaporization for a substance vaporizing [13] [14]. From this definition, the latent heat $\left(Q_{L}\right)$ for a given mass (m) of a considered thermodynamic system substance, necessary for the phase transition is:

$$
Q_{L}=m \lambda
$$

Where, $\lambda$ is the specific latent heat for a particular substance:

$$
\frac{Q_{L}}{m}
$$

In the scientific literature, there are commonly quoted/tabulated and defined specific terms, latent heat of fusion $\left(\lambda_{f}\right)$ and the specific latent heat of vaporization $\left(\lambda_{v}\right)$ to describe a substance of a thermodynamic system. 
Sensible heat and latent heat describe exchanges of heat under conditions prefixed/specified. Therefore, they aren't special forms of energy and aren't described a property of one system, or contained within it, because the heat is defined as the energy transferred spontaneously from a hottest to a coldest thermodinamyc system [15] [16].

Instead, thermal power $(\dot{Q})$ is the amount of heat/thermal energy $(Q)$ transferred per unit time $(t)$. Thermal power (equivalently $P_{t}$ ), as a function of time, is the rate at which work is done, so can be expressed by this equation:

$$
P_{t}=\frac{\mathrm{Q}}{\mathrm{t}}
$$

The standard unit for the rate of heat transferred is the watt $(\mathrm{W})$, defined as one joule per second:

$$
W=\frac{J}{s}
$$

Mpemba effect

The effect is named after Erasto Mpemba, described it in 1963 [17] [18]. The phenomenon is taken to mean "hot water freezes faster than cold", is impossible, if the conditions of the observations are identical, as it would violate the first or second law of thermodynamics.

\section{THOUGHT EXPERIMENT}

It is worth mentioning some hypothesis/principles, for the purpose of thinking through its consequences. The common goal of this thought experiment is to explore the potential consequences of the effect in question, calculated theoretically applying the laws of physics, and carried out to support, refute and validate observations of this research.

Fundamental Proposition: If you take two similar containers with equal volumes of water, one hottest and the other coldest, and put them into a freezer, the coldest water freezes first $\left(T_{\mathrm{f}}=273,15 \mathrm{~K}\right)$.

\section{DEMONSTRATION}

[19] New Scientist recommends starting the experiment with containers/glass at: $35^{\circ} \mathrm{C}$ for hottest water temperature $\left(T_{h}\right)$ and $5{ }^{\circ} \mathrm{C}$ for coldest water temperature $\left(T_{c}\right)$, to maximize the Mpemba effect.

It is highlighted that the hottest water has the following properties:

Temperature $(T)=308,15 \mathrm{~K}$; mass $(\mathrm{m})=137,50 \mathrm{~g}=0,1375 \mathrm{~kg}$; specific heat capacity $(\mathrm{c})=\frac{4,183 \mathrm{~kJ}}{\mathrm{kgK}}$; density $(\rho)=$ $994 \frac{\mathrm{kg}}{\mathrm{m}^{3}}$; specific volume $\left(\mathrm{v}, \rho^{-1}\right)=0,001006 \frac{\mathrm{m}^{3}}{\mathrm{~kg}}=1,006 \frac{\mathrm{dm}^{3}}{\mathrm{~kg}} ;$ volume $(\mathrm{V})=138,30 \mathrm{ml}$.

From these properties, it is possible to calculate the specific heat:

$Q_{s_{h}}=m c \Delta T_{s_{h}}=20,13 \mathrm{~kJ}$

The heat capacity is often affected by many of the state variables that describe the thermodynamic system, among which the temperature, pressure and volume. So, there are actually different measurements of heat capacity. The most commonly used methods for measurement are to hold the thermodynamic system either at constant pressure or at constant volume. In this specific case, the used method for measurement is at constant pressure. Therefore, at constant pressure:

$$
\mathrm{Q}_{\mathrm{sh}_{\mathrm{h}}}=m \Delta h_{s_{h}}
$$


Enthalpy of a thermodynamic system substance describe the transfer of energy in many forms i. e. the heat [20].

The enthalpy $(\mathrm{H})$ of a thermodynamic system is equal to the sum of system's internal energy with the product of its volume and pressure [21] [22].

For isobaric processes, the heat absorbed or released equals the change of enthalpy.

The unit of measurement for enthalpy in the International System of Units (SI) is the joule (J). Whereas, The SI unit for specific enthalpy (h) is joule per kilogram:

$\frac{J}{k g}$

It is worth mentioning/looking at the thermodynamic process using the $\mathrm{p}$-h diagram in Figure 2:

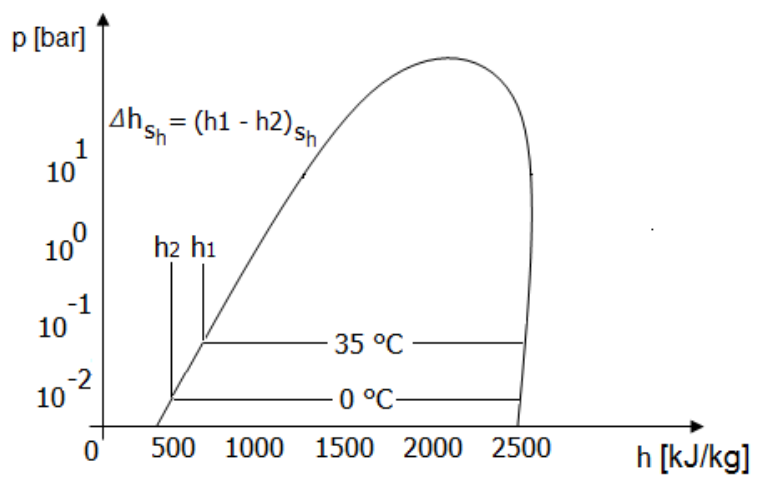

Figure 2: p-h Diagram of Freezing for the Hottest Water

With: $h_{1}=646,70 \frac{\mathrm{kJ}}{\mathrm{kg}}$ and $h_{2}=500,06 \frac{\mathrm{kJ}}{\mathrm{kg}}$. Therefore: $\Delta h_{s_{h}}=146,64 \frac{\mathrm{kJ}}{\mathrm{kg}}=c \Delta T_{s_{h}}$

It is highlighted that the coldest water has the following properties:

Temperature $(\mathrm{T})=278,15 \mathrm{~K}$; mass $(\mathrm{m})=137,50 \mathrm{~g}=0,1375 \mathrm{~kg}$; specific heat capacity $(\mathrm{c})=\frac{4,200 \mathrm{~kJ}}{\mathrm{kgK}} ;$ density $(\rho)=$ $1000 \frac{\mathrm{kg}}{\mathrm{m}^{3}} ;$ specific volume $\left(\mathrm{v}, \rho^{-1}\right)=0,001 \frac{\mathrm{m}^{3}}{\mathrm{~kg}}=1 \frac{\mathrm{dm^{3 }}}{\mathrm{kg}} ;$ volume $(\mathrm{V})=137,50 \mathrm{ml}$

From these properties, it is possible to calculate the specific heat:

$\mathrm{Q}_{\mathrm{s}_{\mathrm{c}}}=m c \Delta T_{s_{c}}=2,89 \mathrm{~kJ}$

Also in this case, the used method for measurement is at constant pressure:

$\mathrm{Q}_{\mathrm{s}_{\mathrm{c}}}=m \Delta h_{s_{c}}$

It is worth mentioning/looking at the thermodynamic process using the $\mathrm{p}$-h diagram in Figure 3: 


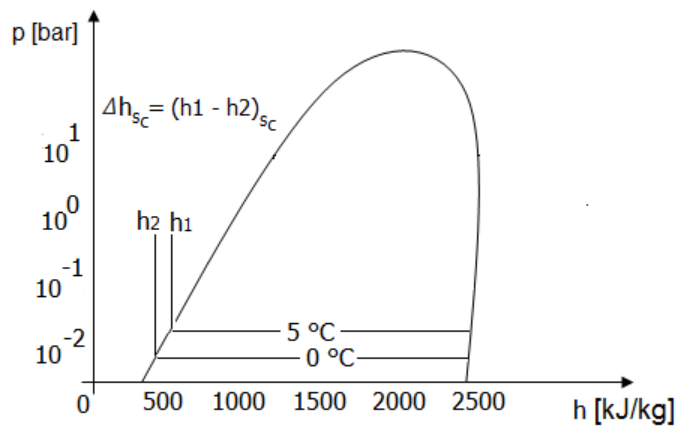

Figure 3: p-h Diagram of Freezing for the Coldest Water

With: $h_{1}=521,12 \frac{\mathrm{kJ}}{\mathrm{kg}}$ and $h_{2}=500,06 \frac{\mathrm{kJ}}{\mathrm{kg}}$. Therefore: $\Delta h_{s_{c}}=21,06 \frac{\mathrm{kJ}}{\mathrm{kg}}=c \Delta T_{s_{c}}$

(Q) This means that the change in enthalpy under such conditions is lower. Enthalpies for chemical substances at constant pressure usually refer to standard state: most commonly 1 bar pressure.

Cooling capacity is the measure of a cooling system's ability to remove heat [23]. The SI units are watts (W). The basic SI unit's equation for deriving cooling capacity is of the form:

$\dot{\mathrm{Q}}=\dot{\mathrm{m} c} \Delta \mathrm{T}$

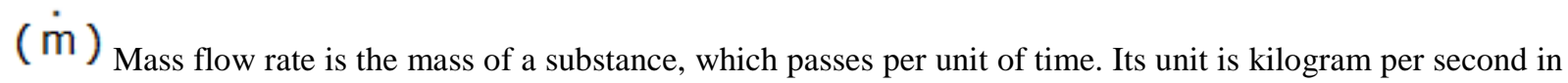
SI units. Sometimes, mass flow rate is termed mass flux or mass current [24].

It is supposed that the two containers with equal volumes of water, one hottest and the other coldest, are placed in a freezer with a predetermined cooling capacity. The cooling time $(\mathrm{t})$ is measured in seconds, and is determined by the following equation:

$$
\mathrm{t}=\frac{\mathrm{Qs}_{\mathrm{s}}}{\dot{\mathrm{Q}}}
$$

It is highlighted that:

$Q_{s_{h}}>Q_{s_{C}} \Rightarrow t_{s_{h}}>t_{s_{C}}$

With:

$$
\mathrm{t}_{\mathrm{s}_{\mathrm{h}}}=\frac{\mathrm{Q}_{\mathrm{s}_{\mathrm{h}}}}{\dot{\mathrm{Q}}}>\mathrm{t}_{\mathrm{s}_{\mathrm{c}}}=\frac{\mathrm{Q}_{\mathrm{s}_{\mathrm{C}}}}{\dot{\mathrm{Q}}}
$$

Therefore, as it turns out, the coldest water freezes first, this is what the author was trying to demonstrate.

Indeed, when $T_{h}$ reach $T_{c}$ :

$T_{c}=T_{h}-\Delta T$ If instead absurdly, let us assume that the Mpemba effect is valid, then:

$t_{s_{h}}<t_{s_{c}}$

Therefore, it is worth to start from the first law of thermodynamics for closed systems, for an infinitesimal 
process:

$d U=\delta Q-\delta L$

Where, $\mathrm{U}$ is internal energy, $\mathrm{L}$ is work and Q is heat [25]. Pressure (p)-volume (V) work by the closed system is defined as:

$d U=\delta Q-p d V$

The first law of thermodynamics is often is expressed in terms of enthalpy:

$d H=\delta Q+V d_{p}$

For an isobaric transformation (constant pressure), the first law of thermodynamics is reduced to:

$\delta Q=d H$

[26] [27] it is remembered that the enthalpy of a thermodynamic system is defined as:

$H=U+p V$

About this, assuming that $t_{s_{h}}<t_{s_{c}}$ then:

$Q_{s_{h}}<Q_{s_{C}} \Rightarrow \Delta h_{s_{h}}<\Delta h_{s_{c}} \Rightarrow \Delta T_{s_{h}}<\Delta T_{s_{c}}$

The final result is an absurd, since the initial hypothesis was:

$\Delta T_{s_{h}}>\Delta T_{s_{C}}$

As a matter of fact, the Mpemba effect violates the fundamental thermodynamic laws [29] [30] [31], because:

$\left(h_{1}-h_{2}\right)_{s h}<c \Delta T_{s_{h}} \Rightarrow \Delta S_{s h}<0$

$\Delta S_{S h}$ introduces the measurement of entropy change [32]. Thermodynamic Entropy change quantifies the entity of some thermal changes, such as heat transfer between systems.

In the International System Unit, the thermodynamic entropy (S) have dimension of energy divided by temperature, and has a unit of joules per kelvin:

$\frac{J}{K}$

The closed systems tend to increasing entropy, that correspond to irreversible change of energy expended as waste heat, limiting the amount of work a system can do [33][34][35][36]. Similarly, for isolated systems, entropy never decreases. This fact has several important consequences in physic science: first, it prohibits free energy machines, defined as "perpetual motion"; and second, it implies the arrow of entropy is directly proportional to the arrow of time. This fact leads to an equation/relation is known as the fundamental thermodynamic relation [37].

This relation is: $d U=T d S-p d V$ 


\section{EXPERIMENTAL RESULTS}

To verify the experiment, carried out a procedure to support, refute and validate the hypothesis/thesis, previously demonstrated. Experiments vary greatly in goal and scale, but always rely on repeatable procedure and logical analysis of the results. To prove experimentally the invalidity of the Mpemba effect, the experiment begins with containers/glass at: $35^{\circ} \mathrm{Cfor}$ hottest water temperature $\left(T_{h}\right)$ and $5{ }^{\circ} \mathrm{C}$ for coldest water temperature $\left(T_{c}\right)$. The experiment should maximize the Mpemba effect [19]. On the contrary, the experimental results will show that the Mpemba effect doesn't occur. The following data were obtained in Figure 4:

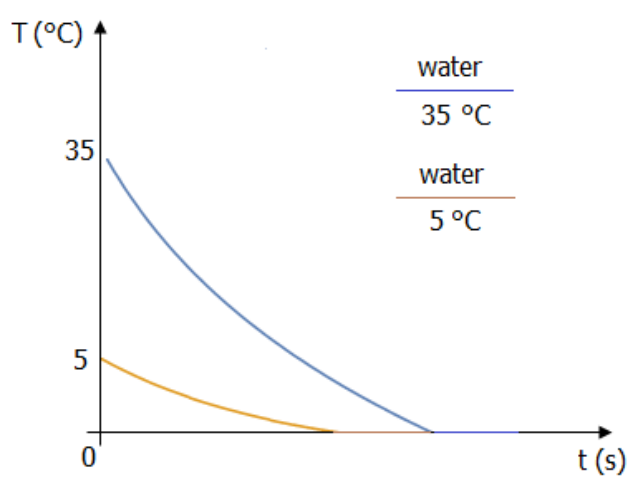

Figure 4: Cooling of Water at $5^{\circ} \mathrm{C}$ and $35{ }^{\circ} \mathrm{C}$ in a Glass

Moreover, the experiment was reproduced in $n=10$ different freezers, with few variations. The materials and methods used are already known [38]. But the result obtained is different, in accordance with the laws of thermodynamics. Some results were obtained in table 1 , observing 3 intervals of 10 minutes:

Table 1: Cooling Time of Water at $5^{\circ} \mathrm{C}$ and $35^{\circ} \mathrm{C}$ in a Glass

\begin{tabular}{|c|c|c|c|}
\hline & $\boldsymbol{t}_{\mathbf{1}}=\mathbf{6 0 0} \boldsymbol{s}$ & $\boldsymbol{t}_{\mathbf{2}}=\mathbf{1 2 0 0} \boldsymbol{s}$ & $\boldsymbol{t}_{\mathbf{3}}=\mathbf{1 8 0 0} \mathrm{s}$ \\
\hline$T_{h}$ & $20,96^{\circ} \mathrm{C}$ & $12,55^{\circ} \mathrm{C}$ & $7,51^{\circ} \mathrm{C}$ \\
\hline$T_{c}$ & $1,95^{\circ} \mathrm{C}$ & $1,1{ }^{\circ} \mathrm{C}$ & $0{ }^{\circ} \mathrm{C}$ \\
\hline
\end{tabular}

As to prove that the coldest water freezes first:

$t_{s_{h}}>t_{s_{c}}$

Similarly, for an infinitesimal process, if:

$T_{h}-T_{c}=d T$

Then:

$t_{s_{h}}-t_{s_{c}}=d t$

The previous solutions/results have a graphical trend of the exponential function (positive semi-axis) when the base $\mathrm{b}$ is between $0-1$ :

$y=c b^{x}$

In which, the argument $\mathrm{x}$ occurs as an exponent and $\mathrm{c}$ occurs as a constant, which depends on the environmental conditions, initial observations, properties and many other factors. 


\section{CONCLUSIONS}

It has been shown that the effect: "hot water can freeze faster than cold water" is not a real/correct effect [39], but it is only the result of trivial operations. The laws of physics are universal and related only to the considered reference system. Moreover, [40] the definition/formulation: "there exists a set of initial parameters, and a pair of temperatures, such that, given two bodies of water identical in these parameters, and differing only in initial uniform temperatures, the hot one will freeze sooner", it is invalid for the following reason: the initial parameters aren't correctly equivalents, because all conditions aren't the same at any time. Similarly, it's obvious that: "hot water freezes faster than cold", particularly if:

$$
\dot{Q}_{h}>\dot{Q}_{c}
$$

and/or:

$m_{h} \ll m_{c} \Rightarrow Q_{h} \ll Q_{c}$

Therefore, only if these different parameters/conditions are satisfied, then there exists a pair of temperatures, such that for the two water samples: the hottest water one will freeze sooner.

\section{REFERENCES}

1. Ball, Philip. Does hot water freeze first? Physics World, pp. 19-26.

2. Aristotle, Meteorology. 4th century BC.

3. Kell, George S. The freezing of hot and cold water. American Journal of Physics, pp. 564-565.

4. CITV Prove It! Series 1 Programme 13.

5. Jin, Jaehyeok, Goddard III, William A. Mechanisms Underlying the Mpemba Effect in Water from Molecular Dynamics Simulations. Journal of Physical Chemistry C., pp. 2622-2629.

6. Lasanta, Antonio; Vega Reyes, Francisco; Prados, Antonio; Santos, Andrés. When the Hotter Cools More Quickly: Mpemba Effect in Granular Fluids. Physical Review Letters.

7. Partington, J. R. An Advanced Treatise on Physical Chemistry, Volume 1, Fundamental Principles. The Properties of Gases, Longmans, Green, and Co., London, pp. 155-157.

8. Prigogine, I., Defay, R. Chemical Thermodynamics, Longmans, Green \& Co, London, pp. 22-23.

9. Adkins, C. J. Equilibrium Thermodynamics, second edition, McGraw-Hill, London, pp. 43-46.

10. Landsberg, P. T. Thermodynamics and Statistical Mechanics, Oxford University, p. 11.

11. International Union of Pure and Applied Chemistry, Physical Chemistry Division. "Quantities, Units and Symbols in Physical Chemistry". Blackwell Sciences. p. 7. The adjective specific before the name of an extensive quantity is often used to mean divided by mass.

12. International Bureau of Weights and Measures, The International System of Units (SI).

13. Perrot, Pierre. A to Z of Thermodynamics. Oxford University Press.

14. Clark, John, O. E. The Essential Dictionary of Science. Barnes \& Noble Books.

15. Robert F. Speyer. Thermal Analysis of Materials. Materials Engineering. Marcel Dekker, Inc. p. 2. 
16. Thomas W. Leland, Jr., G. A. Mansoori, ed., Basic Principles of Classical and Statistical Thermodynamics.

17. Mpemba, Erasto B.; Osborne, Denis G. "Cool?". Physics Education. Institute of Physics. pp. 172-175.

18. Mpemba, Erasto B.; Osborne, Denis G. "The Mpemba effect". Physics Education. Institute of Physics. pp. 410-412.

19. Chown, Marcus. "Revealed: why hot water freezes faster than cold". New Scientist.

20. Thermodynamics: An Engineering Approach by Yunus A. Cengal and Michael A. Boles.

21. "Oxford Living Dictionaries".

22. "IUPAC Gold Book. Enthalpy, H".

23. Boles, Yunus A. Çengel, Michael A. Thermodynamics: an engineering approach. New York: McGraw-Hill. p. 608.

24. Fluid Mechanics, M. Potter, D. C. Wiggart, Schuam's outlines, McGraw Hill.

25. First Law of Thermodynamics". www.grc.nasa.gov

26. Guggenheim, E. A. Thermodynamics. Amsterdam: North-Holland Publishing Company.

27. Zumdahl, Steven S. "Thermochemistry". Chemistry. Cengage Learning. p. 243.

28. Ebbing, Darrel; Gammon, Steven. General Chemistry. Cengage Learning. p. 231.

29. andler, Stanley I. Chemical, biochemical, and engineering thermodynamics. New York: John Wiley \& Sons. p. 91.

30. Simon, Donald A. McQuarrie; John D. (1997). Physical chemistry: a molecular approach (Rev. ed.). Sausalito, Calif.: Univ. Science Books. p. 817.

31. Haynie, Donald, T. (2001). Biological Thermodynamics. Cambridge University.

32. Clausius, Rudolf. Annalen der Physik und Chemie. pp. 353-400.

33. Licker, Mark D. (2004). McGraw-Hill concise encyclopaedia of chemistry.

34. Sethna, James P. (2006). Statistical mechanics: entropy, order parameters, and complexity.

35. Daintith, John. A dictionary of science. Oxford University.

36. de Rosnay, Joel. The Macroscope - a New World View (written by an M. I. T.-trained biochemist). Harper \& Row, Publishers.

37. Schmidt-Rohr, K. "Expansion Work without the External Pressure, and Thermodynamics in Terms of Quasistatic Irreversible Processes". J. Chem. Educ. pp. 402-409.

38. Bregović, Nikola; Mpemba effect from a viewpoint of an experimental physical chemist.

39. Henry C. Burridge, Paul F. Linden. "Questioning the Mpemba effect: hot water does not cool more quickly than cold". Nature.

40. Jeng, Monwhea. "Hot water can freeze faster than cold?!?". American Journal of Physics. 